\title{
Predictors of early rebleeding after endoscopic therapy of first variceal bleeding in liver cirrhosis
}

\author{
Maha Salah Abu Alia', Abdallah Ahmed Elsawy ${ }^{1 *}$ D, Reda Abdelsamaie Elarabawy ${ }^{2}$ and Hegazy Mohamed Hegazy ${ }^{1}$
}

\begin{abstract}
Background: Despite the great advancement in therapeutic modalities for esophageal varices, early variceal rebleeding still occurs at high rates leading to an exaggeration of the morbidity and mortality for cirrhotic patients, so meticulous follow-up with optimum prediction and proper preventive measures for early variceal rebleeding are mandatory for increasing survival of those patients. In this respect, we evaluated the clinical, laboratory, abdominal ultrasound, and endoscopic criteria of variceal cirrhotic patients as possible risk predictors of early variceal rebleeding after endoscopic control of first variceal bleeding. All included patients were followed up blindly for 12 weeks after endoscopic control of bleeding for ascertainment of first variceal rebleeding. The demographic, clinical, laboratory, abdominal ultrasound, and upper gastrointestinal endoscopic criteria were evaluated for all patients at first admission.

Results: By univariate regression analysis, the statistically significant predictors for early variceal rebleeding were serum albumin, serum bilirubin, prothrombin concentration, Child-Pugh score, platelet count, spleen diameter, ascites, portal vein diameter and velocity, variceal size, variceal location, and red color sign. By using multivariate regression analysis, the most independent significant predictors were Child-Pugh score (sig: 0.001 and OR: 1.661), platelets count (sig: 0.000 and OR: 0.956), portal vein velocity (sig: 0.000 and OR: 0.664), variceal grading (sig: 0.000 and OR: 3.964), and variceal red color sign (sig: 0.000 and OR: 4.964). We used the multivariate regression coefficients for the significant predictors to build up early variceal rebleeding risk (EVRR) score with a significant discriminatory performance (AUC: 0.965 and sig: 0.000).
\end{abstract}

Conclusion: Child-Pugh score, platelet count, portal vein velocity, variceal grading, and variceal red color sign are independent risk predictors for early variceal rebleeding after successful control of first variceal bleeding in cirrhotic patients. Our proposed EVRR score could be helpful for the prediction of early variceal rebleeding in cirrhotic patients after endoscopic control of acute variceal bleeding; however, it should be externally validated in large prospective studies.

Keywords: Variceal rebleeding, Predictors, Risk, Cirrhosis, Variceal bleeding

\section{Background}

Despite the recent great advancement in therapeutic modalities for bleeding esophageal varices (EV) in cirrhotic patients, early variceal rebleeding still occurs at higher rates that may reach up to $30-40 \%$ of cases in some reports. This high variceal rebleeding rate could exaggerate its attributed morbidity and mortality burden

\footnotetext{
* Correspondence: abdallahelsawy@hotmail.com

${ }^{1}$ Department of Internal Medicine, Faculty of Medicine, Tanta University, Tanta, Egypt

Full list of author information is available at the end of the article
}

for those cirrhotic patients [1-4], so meticulous followup with an optimum prediction of early rebleeding and proper preventive measures are mandatory for increasing survival of those patients [5-7].

Many predictive risk factors for variceal rebleeding were previously reported with high degree of variability as regards their methodological design, sample size, and results [8-13]. In this respect, we evaluated the most relevant demographic, routine laboratory, and abdomen ultrasound features that are closely related to the pathogenesis and development of esophageal varices (EV) in 
liver cirrhosis and at the same time the endoscopic variceal criteria as well as the type of endoscopic modality of variceal bleeding control either endoscopic band ligation (EBL) or endoscopic injection therapy (EIT) as possible risk predictors of early rebleeding after endoscopic control of first variceal bleeding. In our study, we tried to derive a new prediction score for variceal rebleeding in our cirrhotic patients.

\section{Methods}

\section{Study design and source of data}

This is a prospective predictor cohort study that was conducted in accordance to the TRIPOD Statement (Transparent Reporting of a multivariable prediction model for Individual Prognosis Or Diagnosis) [14]. It was conducted at the emergency endoscopic unit of the internal medicine department at our university hospitals (a tertiary hospital). All participants were consecutively enrolled in the period from August 2019 to April 2020, and they were followed up for 12 weeks after endoscopic successful bleeding control.

\section{Participants}

Five hundred and sixty-two patients were consecutively presented to our emergency endoscopic unit by upper gastrointestinal bleeding (hematemesis and/or melena); all patients were resuscitated and evaluated using upper gastrointestinal endoscopy for the study eligibility criteria before enrollment. These eligibility criteria included proved cirrhotic patients who were consecutively presented by first variceal bleeding that was controlled well by esophagogastroduodenoscope (EGD).

The eligibility criteria were fulfilled in 412 patients who were consecutively presented by cirrhosis and first variceal bleeding that was successfully controlled either by endoscopic band ligation (EBL) or endoscopic injection therapy (EIT). One hundred and fifty patients were excluded before enrollment, 30 patients were excluded due to non-variceal upper GIT bleeding, 20 patients were excluded as they were non-cirrhotic, 60 patients were excluded as they had a history of endoscopic treatment for esophageal varices (EV), 10 patients were excluded due to hepatocellular carcinomas (HCC) or malignant liver metastasis, 10 patients were excluded due to portal vein thrombosis, 6 patients were excluded due to history of splenectomy, 10 patients were excluded as the bleeding source was isolated gastric varices, and 4 patients were excluded as the bleeding was failed to be controlled endoscopically.

All included patients were blindly followed up for 12 weeks after endoscopic control of bleeding for ascertainment of possible variceal rebleeding as our endpoint; the variceal rebleeding was diagnosed according to Baveno criteria by reappearance of hematemesis and/or melena or requirement for $>2$ units of packed red blood cells within $24 \mathrm{~h}$ with hemodynamic instability and was confirmed by upper gastrointestinal (GIT) endoscopy [15]. All these are illustrated in the participant's flowchart (Fig. 1).

\section{Evaluated predictors}

The following base-line criteria were evaluated for all included patients at first admission: demographic criteria (age and sex), clinical presentations (hematemesis and/ or melena), abdominal ultrasound criteria (spleen diameter, portal vein diameter (PVD), portal vein velocity (PVV) and ascites), routine laboratory criteria (AST, ALT, serum albumin, serum bilirubin, prothrombin concentration (PC), serum creatinine, platelet count, and hemoglobin), and upper GIT endoscopic criteria which were graded according to the Japanese Research Society for Portal Hypertension (variceal form, variceal location, and red color signs) [16], as well as endoscopic modality of variceal bleeding control (EBL or EIT).

\section{Sample size estimation}

Sample size estimation was statistically based on the previously reported incidence rate of early variceal rebleeding that ranges from 30 to $40 \%$, the sample size calculation assumed that the confidence interval was 95\% and the estimation error was 0.05 .

\section{Statistical analysis methods}

We used IBM SPSS, version 23 statistic software (IBM, NY, USA) for both summarization and statistical analysis of our collected data. The median with (minimum to maximum) was calculated for all quantitative data as it was abnormally distributed; however, all qualitative data were tabulated as frequency and relative frequency.

The pre-selection of our predictors was based on wellconducted previous reports, clinical reasoning, and on univariate logistic regression analysis of our evaluated predictors. Logistic regression analysis was performed to find out the best predictors of early variceal rebleeding. Univariate logistic regression analysis was done first for each predictor to identify the significant predictor with its unadjusted hazard ratio (OR), and then the most independent significant predictors were evaluated using the multivariable logistic regression analysis by entering all the previously identified significant predictors simultaneously with a stepwise backward strategy.

The regression coefficients of the most independent significant predictors-that were identified in multivariate regression analysis-were used to derive our predicted risk score. Receiver operating characteristic (ROC) curves were calculated for our predictor score and the area under the ROC curve (AUC) was computed. The new predictor score was graded to 3 risk 


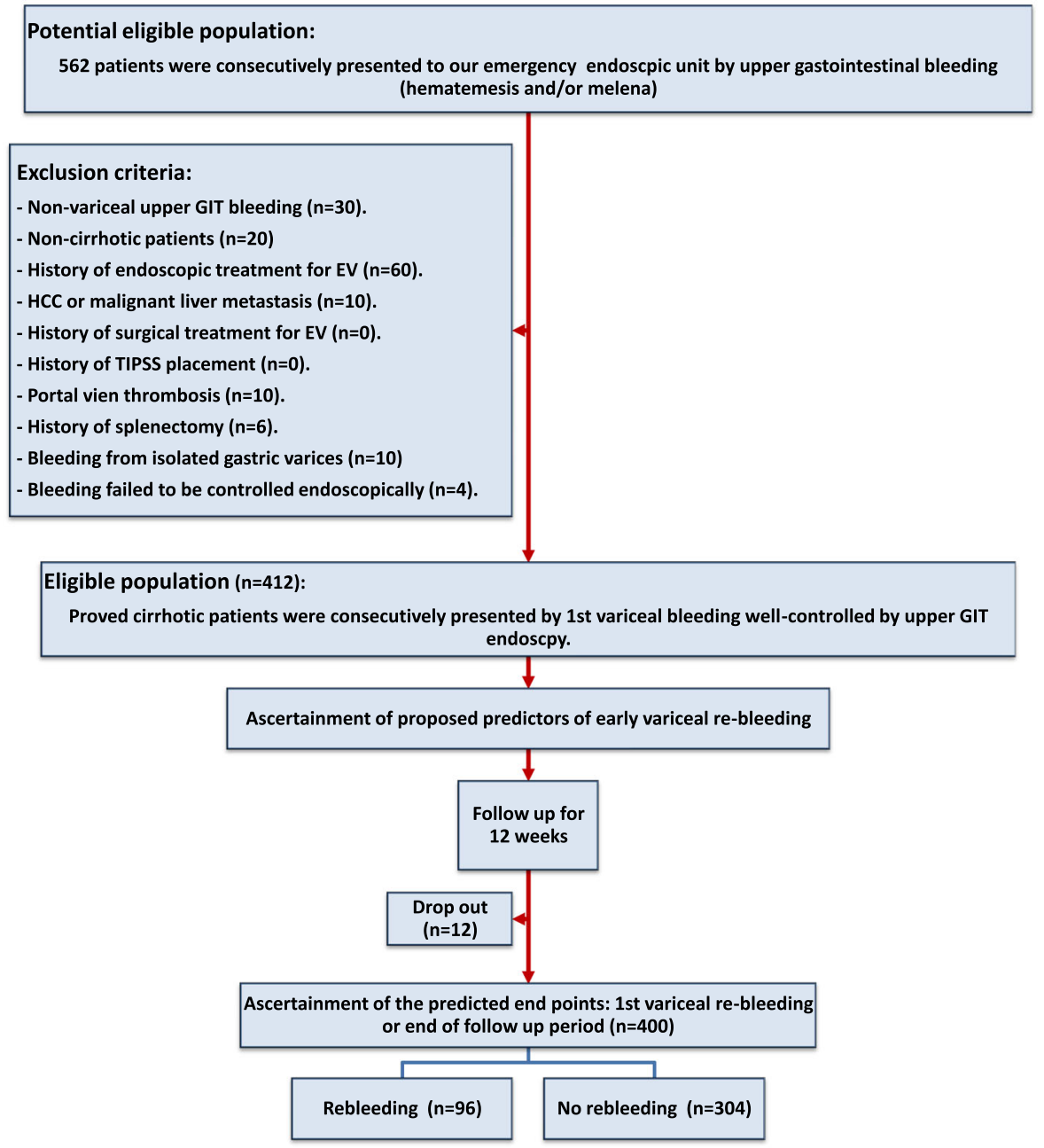

Fig. 1 Participants' flowchart

groups: low, medium, and high risk by using cutoff points along its scale; the 1st cutoff point was selected to rule in the outcome with the highest specificity and highest $\mathrm{LR}+$, the 2nd cutoff point was selected to rule out the outcome with the highest sensitivity and lowest LR-, and then the hazard distribution in-between different grades was illustrated using Kaplan-Meier method and was analyzed using log-rank test. P values less than 0.05 were considered statistically significant.

\section{Results}

Table 1 of our results shows participants' demographic criteria, the different clinical presentations of acute upper GIT bleeding, the most relevant, laboratory and abdomen ultrasound features that are closely related to pathogenesis and development of EV in liver cirrhosis, the endoscopic variceal criteria, and the type of endoscopic modality of variceal bleeding control. The rebleeding was ascertained in $96(24 \%)$ of our patients during the 12-week follow-up period after control of the first variceal bleeding.

Table 2 of our results shows the univariate logistic regression analysis for our studied proposed predictors for early variceal rebleeding. The statistically significant laboratory predictors were serum albumin, serum bilirubin, PC, Child-Pugh score, and platelets. The statistically significant abdominal ultrasound parameters were spleen diameter, ascites, PVD, and PVV. The statistically significant endoscopic variceal criteria were variceal form, variceal location, and red color sign.

All the previously significant predictors were evaluated simultaneously using multivariate logistic regression analysis to identify the most independent significant predictors for early variceal rebleeding as illustrated in Table 3. The most independent significant predictors were Child-Pugh score, platelets, PVV, variceal form, and variceal red color sign. We used the multivariate logistic regression coefficients that are illustrated in Table 
Table 1 Main evaluated participants' criteria

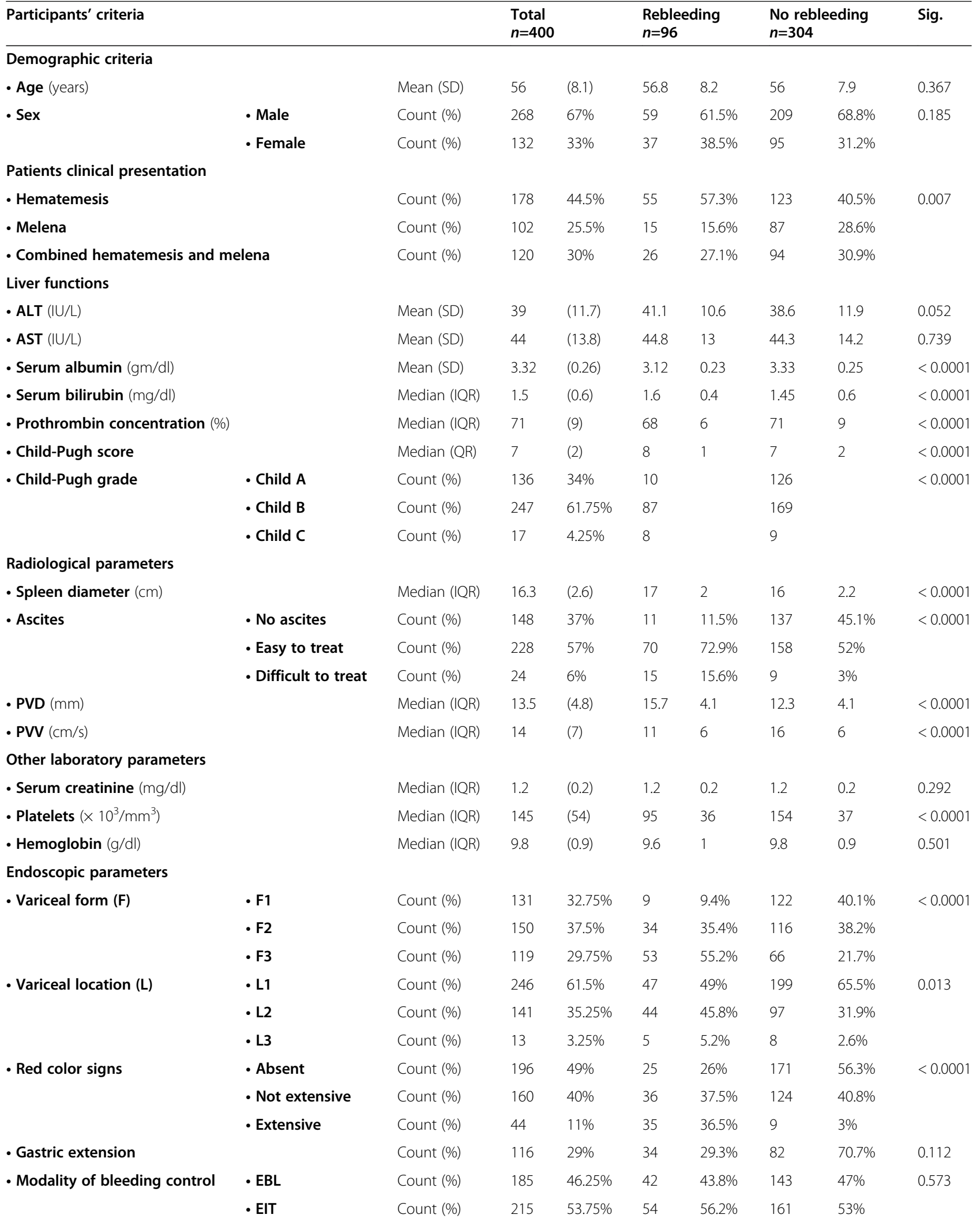


Table 2 Univariate logistic regression analysis for the evaluated predictors

\begin{tabular}{|c|c|c|c|c|c|c|c|c|}
\hline \multirow[t]{2}{*}{ Participants' criteria } & \multirow[t]{2}{*}{ Constant } & \multirow[t]{2}{*}{ B } & \multirow[t]{2}{*}{ SE } & \multirow[t]{2}{*}{ Wald } & \multirow[t]{2}{*}{ Sig. } & \multirow[t]{2}{*}{$\operatorname{EXP}(B)$} & \multicolumn{2}{|l|}{$95 \% \mathrm{Cl}$} \\
\hline & & & & & & & Lower & Upper \\
\hline \multicolumn{9}{|l|}{ Demographic criteria } \\
\hline - Age (years) & -1.901 & 0.13 & 0.015 & 0.817 & 0.366 & 1.013 & 0.985 & 1.043 \\
\hline - Sex & 0.943 & -0.322 & 0.243 & 1.747 & 0.186 & 0.725 & 0.515 & 1.171 \\
\hline \multicolumn{9}{|l|}{ Liver functions } \\
\hline • ALT (IU/L) & -1.850 & 0.018 & 0.010 & 3.286 & 0.07 & 1.018 & 0.999 & 1.037 \\
\hline • AST (IU/L) & 0.003 & 0.002 & 0.008 & 0.102 & 0.749 & 1.003 & 0.986 & 1.019 \\
\hline - Serum albumin (gm/dl) & 9.496 & -3.292 & 0.530 & 38.585 & $<0.0001$ & 0.037 & 0.013 & 0.105 \\
\hline - Serum bilirubin (mg/dl) & -2.175 & 0.635 & 0.222 & 8.199 & 0.004 & 1.887 & 1.222 & 2.914 \\
\hline - Prothrombin concentration (\%) & 8.908 & -0.145 & 0.024 & 36.851 & $<0.0001$ & 0.865 & 0.825 & 0.907 \\
\hline - Child-Pugh score & -4.451 & 0.455 & 0.089 & 26.242 & $<0.0001$ & 1.577 & 1.325 & 1.877 \\
\hline \multicolumn{9}{|l|}{ Radiological parameters } \\
\hline - Spleen diameter (cm) & -4.904 & 0.225 & 0.067 & 11.315 & 0.001 & 1.253 & 1.099 & 1.428 \\
\hline - Ascites & -3.986 & 1.564 & 0.251 & 38.806 & $<0.0001$ & 4.776 & 2.920 & 7.811 \\
\hline - PVD (mm) & -6.596 & 0.378 & 0.051 & 55.551 & $<0.0001$ & 1.459 & 1.321 & 1.612 \\
\hline - PVV $(\mathrm{cm} / \mathrm{s})$ & 4.612 & -0.449 & 0.050 & 79.876 & $<0.0001$ & 0.638 & 0.578 & 0.704 \\
\hline \multicolumn{9}{|l|}{ Other laboratory parameters } \\
\hline - Serum creatinine (mg/dl) & -1.874 & 0.598 & 0.787 & 0.578 & 0.447 & 1.819 & 0.389 & 8.514 \\
\hline - Platelets $\left(\times 10^{3} / \mathrm{mm}^{3}\right)$ & 5.216 & -0.051 & 0.005 & 94.666 & $<0.0001$ & 0.950 & 0.940 & 0.960 \\
\hline - Hemoglobin (g/dl) & -0.140 & -0.103 & 0.178 & 0.338 & 0.502 & 0.561 & 0.636 & 1.278 \\
\hline \multicolumn{9}{|l|}{ Endoscopic parameters } \\
\hline - Variceal form (F) & -3.614 & 1.146 & 0.175 & 42.816 & $<0.0001$ & 3.144 & 2231 & 4.431 \\
\hline - Variceal Location (L) & -2.013 & 0.588 & 0.203 & 8.384 & 0.004 & 1.801 & 1.209 & 2.681 \\
\hline - Red color sign & -2.258 & 1.436 & 0.196 & 53.780 & $<0.0001$ & 4.205 & 2.865 & 6.173 \\
\hline - Gastric extension & -1.276 & 0.395 & 0.249 & 2.509 & 0.113 & 1.485 & 0.910 & 2.421 \\
\hline - Modality of bleeding control & -1.225 & 0.133 & 0.236 & 0.317 & 0.573 & 1.142 & 0.720 & 1.812 \\
\hline
\end{tabular}

ALT alanine aminotransferase, AST aspartate aminotransferase, $P V D$ portal vein diameter, PVV portal vein velocity

3 for the most independent significant predictors to build up a predictor model for early variceal rebleeding using the following equation of the predicted probability: $\exp \left[1.826+0.508 *\right.$ Child-Pugh score $-0.045^{*}$ PLT $0.409 \% \mathrm{PVV}+1.242 *$ variceal form $+1.602 *$ variceal red color sign $] /(1+\exp [1.826+0.508 *$ Child-Pugh score $0.045 *$ PLT $-0.409 *$ PVV + 1.242*variceal form $+1.602 *$ variceal red color sign]), where PLT count was in $10^{3} / \mathrm{ml}$ and $\mathrm{PVV}$ was in $\mathrm{cm} / \mathrm{s}$. We named this predictor model as early variceal rebleeding risk score (EVRR score).

Table 3 Multivariable regression analysis for the evaluated predictors

\begin{tabular}{|c|c|c|c|c|c|c|c|}
\hline \multirow[t]{2}{*}{ Participants' criteria } & \multirow[t]{2}{*}{ B } & \multirow[t]{2}{*}{ SE } & \multirow[t]{2}{*}{ Wald } & \multirow[t]{2}{*}{ Sig. } & \multirow[t]{2}{*}{$\operatorname{EXP}(B)$} & \multicolumn{2}{|l|}{$95 \% \mathrm{Cl}$} \\
\hline & & & & & & Lower & Upper \\
\hline - Child-Pugh score & 0.508 & 0.159 & 10.226 & 0.001 & 1.661 & 1.2117 & 2.268 \\
\hline - Platelets $\left(\times 10^{3} / \mathrm{mm}^{3}\right)$ & -0.045 & 0.008 & 33.362 & $<0.0001$ & 0.956 & 0.941 & 0.970 \\
\hline - PVV (cm/s) & -0.409 & 0.080 & 26.108 & $<0.0001$ & 0.664 & 0.568 & 0.777 \\
\hline - Variceal form & 1.242 & 0.287 & 18.728 & $<0.0001$ & 3.964 & 1.973 & 6.077 \\
\hline - Red color sign & 1.602 & 0.309 & 26.950 & $<0.0001$ & 4.964 & 2.711 & 9.089 \\
\hline Constant & 1.826 & & & & & & \\
\hline
\end{tabular}

PVV portal vein velocity

The predicted probability $=\exp \left[1.826+0.508^{*}\right.$ Child-Pugh score $-0.045^{*} \mathrm{PLT}-0.409 * \mathrm{PVV}+1.242^{*}$ variceal form $+1.602^{*} \mathrm{variceal}$ red color sign $] /(1+\exp [1.826+$ $0.508^{*}$ Child-Pugh score $-0.045^{*} \mathrm{PLT}-0.409^{*} \mathrm{PVV}+1.242^{*}$ variceal form $+1.602 *$ variceal red color sign])

Variceal forms were numerically coded ( 1 for F1, 2 for F2, and 3 for F3) and red color signs were numerically coded ( 0 for absent, 1 for non-extensive, and 2 for extensive) 
Table 4 and Fig. 2 of our results illustrate the discriminatory performance of our proposed EVRR score using the receiver operating characteristics that identified two cutoff points $(\leq 0.10$ and $\geq 0.90)$; the 1 st cutoff point was selected to rule out the possibility of occurrence of early variceal rebleeding for values equal or below it with its sensitivity, specificity, LR+, and LR- were $98 \%, 80 \%$, 4.96 , and 0.03 , respectively, and the 2 nd cutoff point was selected to rule in the possibility of occurrence of early variceal rebleeding for values equal or above it with its sensitivity, specificity, LR+, and LR- were 43\%, 99.7\%, 129.8 , and 0.57 , respectively (AUC:0.965 and sig $<0.0001$ ).

We graded the EVRR score to 3 grades using the previous two cutoff points for risk stratification: values $\leq$ 0.10 to identify EVRR grade 1 with mild risk, values $\geq$ 0.90 to identify EVRR grade 3 with high risk, and EVRR grade 2 with moderate risk for the remaining values. The crosstabulation between different risk scores and rebleeding distribution was illustrated in Table 5, the rebleeding free survival function of the different risk grades of EVRR score was illustrated using the KaplanMeier curve, and their pairwise comparisons were analyzed using log-rank test as illustrated in Fig. 3, with statistically significant difference between the different risk grades $($ sig $<0.0001)$.

\section{Discussion}

The high variceal rebleeding rate could exaggerate the morbidity and mortality burden on cirrhotic patients, so meticulous follow-up with an optimum prediction of rebleeding with proper preventive measures are mandatory for those patients to mitigate this devastating complication and increase survival [1-7]. This assumption of the deleterious effects of variceal rebleeding was taken as a rationale by many studies for the exploration of the possible predictor factors for this risk with wide variable results [8-13]. In this respect, we tried searching for the most independent predictor factors that may increase the hazard of early variceal rebleeding in cirrhotic patients after successful endoscopic control of the first episode of variceal bleeding. In our study, we took into consideration the most relevant routine laboratory and radiological criteria that are closely related to pathogenesis and development of EV in liver cirrhosis, as well as the endoscopic variceal criteria as regards its severity and bleeding risk signs and at the same time the type of endoscopic modality of variceal bleeding control.

As regards the underlying baseline liver functions, we found that higher Child-Pugh score, hypo-albuminemia, hyper-bilirubinemia, and lower levels of prothrombin concentration were significant predictors for early variceal rebleeding; however, the Child-Pugh score was found to be the most independent significant predictor factor using multivariate analysis ( $\mathrm{sig}=0.001$ and $\mathrm{OR}=$ 1.661$,$) . In accordance with our results, many reports$ identified that early variceal rebleeding rate significantly increases in higher Child-Pugh scores than lower scores $[17,18]$. We could explain this finding, as the ChildPugh score is a surrogate parameter for the underlying liver cell functions that are deteriorated in accordance to the progression of the underlying liver cirrhosis which is considered as the leading cause of portal hypertension [5, 7].

After multivariate analysis of other laboratory criteria for our participants, we found that thrombocytopenia was the only independent significant predictor for variceal rebleeding. This finding is confirmed by the results of previous reports that identified the possible role of thrombocytopenia in the prediction of portal hypertension and esophageal varices in patients with liver cirrhosis $[19,20]$.

The univariate regression analysis of the baseline radiological criteria of our participants identified that splenomegaly, increased ascites, increased PVD, and decreased PVV were significantly associated with increased risk of early variceal rebleeding. However, by multivariate analysis, we found that PVV was the only independent significant predictor for the risk of variceal rebleeding. The reliability of PVV as a non-invasive tool for the prediction of esophageal varices in cirrhotic patients was confirmed previously in many reports [21, 22]. Consequently, we could suggest that PVV may be used not only in the prediction of esophageal varices in cirrhotic patients but also in the prediction of early variceal rebleeding in those patients. However, to our knowledge, there were no studies that discussed the correlation between PVV and the risk of early variceal rebleeding of esophageal varices.

As regards the endoscopic variceal criteria of our participants, the univariate regression analysis showed that

Table 4 Receiver operating characteristics of the proposed prediction model

\begin{tabular}{|c|c|c|c|c|c|c|c|c|c|}
\hline & \multirow[t]{2}{*}{ Role } & \multirow[t]{2}{*}{ Cutoff } & \multirow[t]{2}{*}{ Sensitivity } & \multirow[t]{2}{*}{ Specificity } & \multirow[t]{2}{*}{ LR+ } & \multirow{2}{*}{$\begin{array}{l}\text { LR } \\
-\end{array}$} & \multicolumn{3}{|l|}{ AUC } \\
\hline & & & & & & & Value & $(95 \% \mathrm{Cl})$ & Sig. \\
\hline \multirow[t]{2}{*}{ Proposed model } & Rule out & $\leq 0.10$ & $98 \%$ & $80 \%$ & 4.96 & 0.03 & 0.965 & $(0.948-0.981)$ & $<0.0001$ \\
\hline & Rule in & $\geq 0.90$ & $43 \%$ & $99.7 \%$ & 129.8 & 0.57 & & & \\
\hline
\end{tabular}

$L R$ likelihood ratio, AUC area under the curve

Risk grading of the proposed prediction model: grade 1 (Low risk) if the predicted probability $\leq 10 \%$; grade 3 (high risk) if the predicted probability $\geq 90 \%$, and grade 2 (moderate risk) for other values 


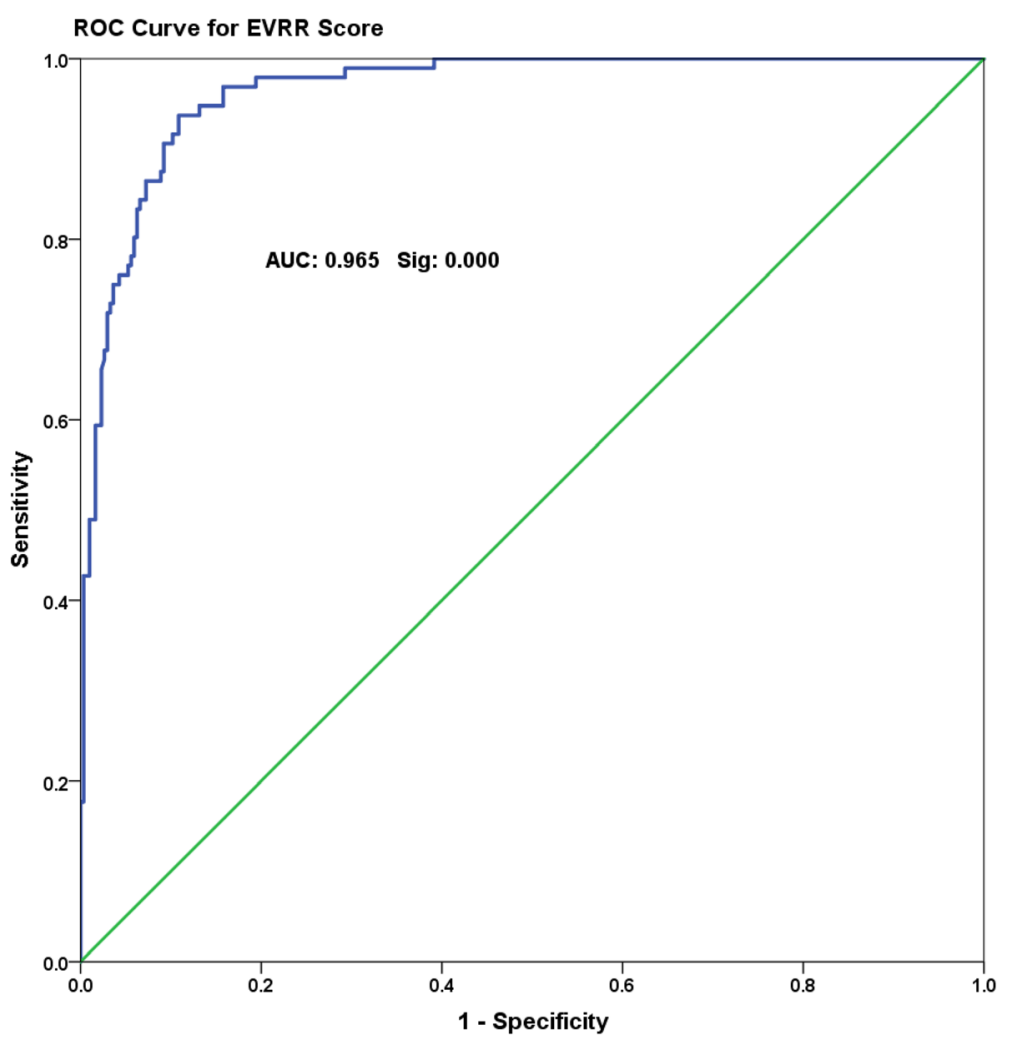

Fig. 2 ROC curve for early variceal rebleeding risk score

variceal grading, variceal location, and red color signs were significantly associated with the risk of rebleeding. However, the multivariate regression analysis identified that the most independent significant endoscopic variceal criteria were EV grading and variceal red color sign. These results are in agreement with many previous reports that found a significant association of variceal rebleeding with variceal size [23-25] and variceal red color sign or nipple sign [24-26].

In summary, after univariate and multivariate analysis of all our potential predictors for variceal rebleeding, we found that the only independent significant predictors were higher levels of the Child-Pugh score, thrombocytopenia, decreased PVV, larger variceal size, and the presence of variceal red color risk sign. All of these five independent significant predictors are related to pathophysiology or the complications of portal hypertension and EV in cirrhotic patients $[7,27]$.

We used the multivariate regression coefficients of those five independent significant predictors to derive a new early variceal rebleeding risk (EVRR) score that revealed a significant discriminatory performance, and two cutoff points $(\leq 0.10$ and $\geq 0.90)$ were identified; the 1 st cutoff point was selected to rule out the possibility of

Table $\mathbf{5}$ Variceal rebleeding distribution in accordance to a risk score of the proposed model

\begin{tabular}{|c|c|c|c|c|c|}
\hline & & & \multicolumn{2}{|c|}{ Rebleeding } & \multirow[t]{2}{*}{ Total } \\
\hline & & & Absent & Present & \\
\hline \multirow[t]{6}{*}{ Risk group } & Mild Risk & Count & 245 & 2 & 247 \\
\hline & & $\%$ within Risk_group & $99.2 \%$ & $0.8 \%$ & $100.0 \%$ \\
\hline & Moderate risk & Count & 58 & 54 & 112 \\
\hline & & $\%$ within Risk_group & $51.8 \%$ & $48.2 \%$ & $100.0 \%$ \\
\hline & High risk & Count & 1 & 40 & 41 \\
\hline & & $\%$ within Risk_group & $2.4 \%$ & $97.6 \%$ & $100.0 \%$ \\
\hline \multirow[t]{2}{*}{ Total } & & Count & 304 & 96 & 400 \\
\hline & & $\%$ within Risk_group & $76.0 \%$ & $24.0 \%$ & $100.0 \%$ \\
\hline
\end{tabular}




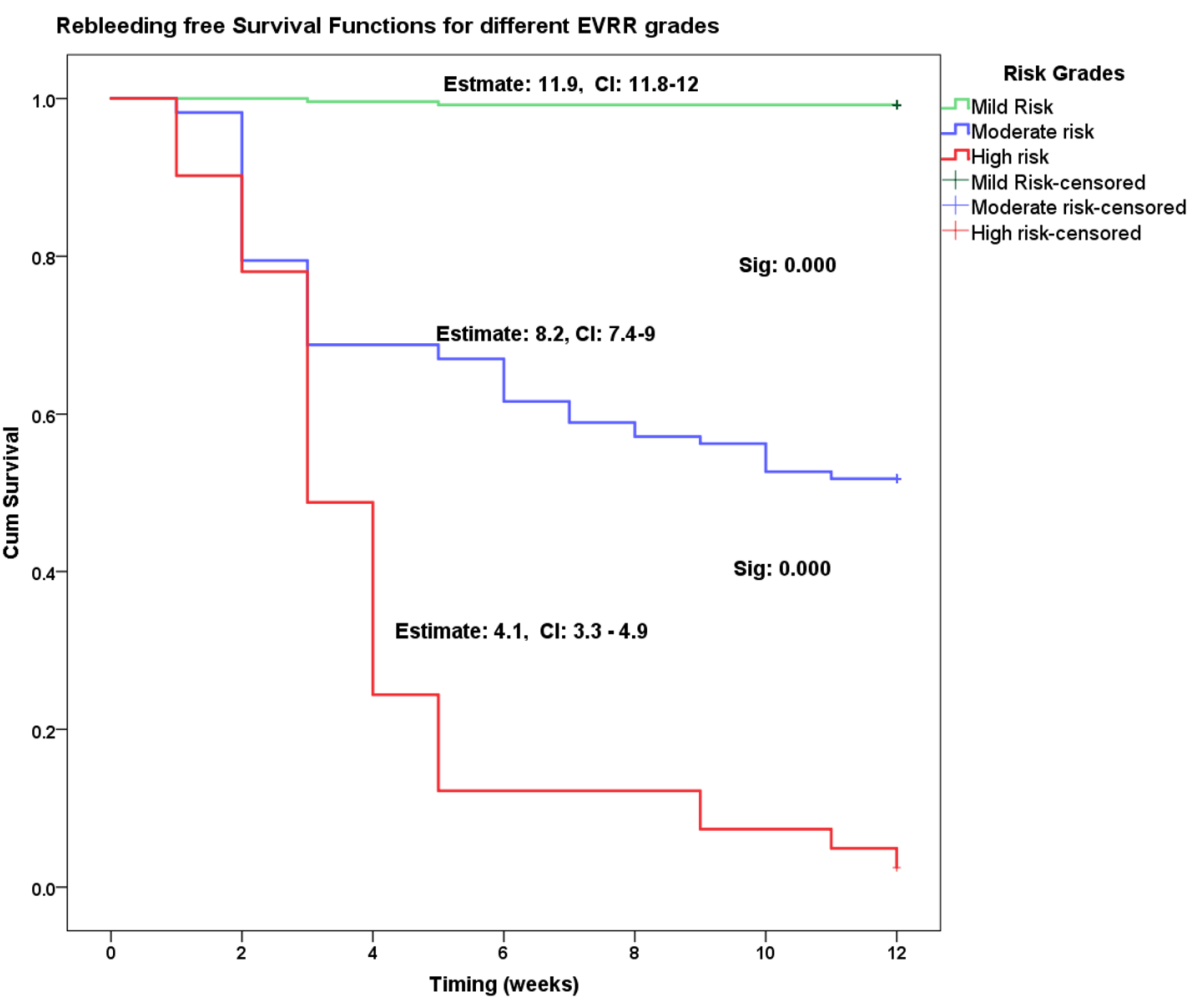

Fig. 3 Rebleeding free survival function for different grades of early variceal rebleeding risk score

occurrence of early variceal rebleeding for values equal or below it and the 2nd cutoff point was selected to rule in the possibility of occurrence of early variceal rebleeding for values equal or above it. We graded the EVRR score to 3 grades using those two cutoff points for risk stratification: values $\leq 0.10$ to identify EVRR grade 1 with mild risk, values $\geq 0.90$ to identify EVRR grade 3 with high risk, and EVRR grade 2 with moderate risk for remaining values, the pairwise comparisons of rebleeding free survival function between the different risk grades of EVRR identified statistically significant difference. However, this proposed score should be externally validated later in large prospective studies.

We found some aspects of limitations in our study, one of them is the single center enrollment that may limit the study generalizability, and the presence of more than one operator for both endoscopy and abdomen ultrasound that may increase the inter-observer variability in values of predictors; however, this limitation was mitigated through their highly trained experience and using of advanced equipment. Other aspects of limitations, as we did not take into consideration the medical treatment that may be prescribed for variceal patients after endoscopy and during the follow-up data like nonselective beta-blockers and proton pump inhibitors, however, these medical treatments were prescribed to most of our patients.

\section{Conclusion}

We concluded that the Child-Pugh score, platelet count, PVV, EV grading, and variceal red color sign are the most independent significant risk predictors for early variceal rebleeding after endoscopic control of first variceal bleeding in cirrhotic patients. Our proposed EVRR score could be helpful for the prediction of early variceal rebleeding in cirrhotic patients after endoscopic control of acute variceal bleeding; however, it should be externally validated in large prospective studies.

\section{Abbreviations}

ALT: Aspartate aminotransferase; AST: Alanine aminotransferase; AUC: Area under the curve; EBL: Endoscopic band ligation; EIT: Endoscopic injection therapy; EV: Esophageal varices; EVRR: Early variceal rebleeding risk; GIT: Gastrointestinal tract; HCC: Hepatocellular carcinoma; LR: Likelihood ratio; OR: Odds ratio; PC: Prothrombin concentration; PLT: Platelets; PVD: Portal vein diameter; PW: Portal vein velocity; Sig: Significance; TRIPOD: Transparent Reporting of a multivariable prediction model for Individual Prognosis Or Diagnosis

\section{Acknowledgements}

We would like to thank all staff members in the Gastroenterology Unit of the Internal Medicine Department at Tanta University Hospital that assisted us in this study.

\section{Authors' contributions}

All authors read and approved the final manuscript, according to the following respective roles of each author. MSA shared in study conception and design, data collection, and data interpretation. AAE shared in study conception and design, data collection, data analysis, data interpretation, and as a corresponding author. RAE shared in study conception and design, 
data collection, and data interpretation. $\mathrm{HMH}$ shared in study conception and design, data collection, and data interpretation.

\section{Funding}

Not applicable

\section{Availability of data and materials}

The datasets used and/or analyzed during the current study are available from the corresponding author on reasonable request.

\section{Declarations}

\section{Ethics approval and consent to participate}

The present study was conducted in accordance with the Declaration of Helsinki. The study protocol was approved by the ethics committee of the Tanta Faculty of Medicine (No: 33264/07/19). All patients provided written informed consent. The results of the research were used only in scientific purposes and not in any other aims.

\section{Consent for publication}

Not applicable

\section{Competing interests}

The authors declare that they have no competing interests.

\section{Author details}

${ }^{1}$ Department of Internal Medicine, Faculty of Medicine, Tanta University, Tanta, Egypt. ${ }^{2}$ Department of Diagnostic Radiology, Faculty of Medicine, Tanta University, Tanta, Egypt.

Received: 11 February 2021 Accepted: 2 June 2021

Published online: 14 June 2021

\section{References}

1. Del Olmo JA, Peña A, Serra MA, Wassel AH, Benages A, Rodrigo JM (2000) Predictors of morbidity and mortality after the first episode of upper gastrointestinal bleeding in liver cirrhosis. J Hepatol 32(1):19-24. https://doi. org/10.1016/S0168-8278(01)68827-5

2. Reverter E, Tandon P, Augustin S, Turon F, Casu S, Bastiampillai R, Keough A, Llop E, González A, Seijo S, Berzigotti A, Ma M, Genescà J, Bosch J, GarcíaPagán JC, Abraldes JG (2014) A MELD-based model to determine risk of mortality among patients with acute variceal bleeding. Gastroenterology 146(2):412-419. https://doi.org/10.1053/j.gastro.2013.10.018

3. Sempere L, Palazón JM, Sánchez-Payá J, Pascual S, de Madaria E, Poveda MJ, Carnicer F, Zapater P, Pérez-Mateo M (2009) Assessing the short- and longterm prognosis of patients with cirrhosis and acute variceal bleeding. Rev Esp Enferm Dig 101(4):236-248

4. Chen WT, Lin CY, Sheen IS, Huang CW, Lin TN, Lin CJ, Jeng WJ, Huang CH, Ho YP, Chiu CT (2011) MELD score can predict early mortality in patients with rebleeding after band ligation for variceal bleeding. World J Gastroenterol 17(16):2120-2125. https://doi.org/10.3748/wjg.v17.i16.2120

5. Bari K, Garcia-Tsao G (2012) Treatment of portal hypertension. World J Gastroenterol 18(11):1166-1175. https://doi.org/10.3748/wjg.v18.i11.1166

6. Lo GH (2006) Prevention of esophageal variceal rebleeding. J Chin Med Assoc 69(12):553-560. https://doi.org/10.1016/S1726-4901(09)70328-4

7. Garcia-Tsao G, Sanyal AJ, Grace ND, Carey W (2007) Prevention and management of gastroesophageal varices and variceal hemorrhage in cirrhosis. Hepatology 46(3):922-938. https://doi.org/10.1002/hep.21907

8. Hunaysh AY (2016) Prediction of early rebleeding and mortality after acute esophageal variceal hemorrhage among Yemeni patients in major hospitals—Sana'a. Open Journal of Gastroenterology 6(08):214-227. https:// doi.org/10.4236/ojgas.2016.68027

9. Krige JE, Kotze UK, Distiller G, Shaw JM, Bornman PC (2009) Predictive factors for rebleeding and death in alcoholic cirrhotic patients with acute variceal bleeding: a multivariate analysis. World J Surg 33(10):2127-2135. https://doi.org/10.1007/s00268-009-0172-6

10. Lee SW, Lee TY, Chang CS (2009) Independent factors associated with recurrent bleeding in cirrhotic patients with esophageal variceal hemorrhage. Dig Dis Sci 54(5):1128-1134. https://doi.org/10.1007/s10620008-0454-0
11. Hunter SS, Hamdy S (2013) Predictors of early re-bleeding and mortality after acute variceal haemorrhage. Arab J Gastroenterol 14(2):63-67. https:// doi.org/10.1016/j.ajg.2013.05.001

12. Wang MT, Liu T, Ma XQ, He J (2011) Prognostic factors associated with rebleeding in cirrhotic inpatients complicated with esophageal variceal bleeding. Chin Med J 124(10):1493-1497

13. Vanbiervliet G, Giudicelli-Bornard S, Piche T, Berthier F, Gelsi E, Filippi J, Anty R, Arab K, Huet PM, Hebuterne X, Tran A (2010) Predictive factors of bleeding related to post-banding ulcer following endoscopic variceal ligation in cirrhotic patients: a case-control study. Aliment Pharmacol Ther 32(2):225-232. https://doi.org/10.1111/j.1365-2036.2010.04331.x

14. Moons KGM, Altman DG, Reitsma JB, loannidis JPA, Macaskill P, Ewout W et al (2001) Transparent Reporting of a multivariable prediction model for Individual Prognosis Or Diagnosis (TRIPOD): Explanation and Elaboration. Ann Intern Med 162:W1-W73

15. de Franchis R (2010) Revising consensus in portal hypertension: report of the Baveno $V$ consensus workshop on methodology of diagnosis and therapy in portal hypertension. J Hepatol 53(4):762-768. https://doi.org/10.1 016/j.jhep.2010.06.004

16. Japanese Research Society for Portal Hypertension and Inokuchi K (1980) The general rules for recording endoscopic findings on esophageal varices. Jpn J Surg 10(1):84-87. https://doi.org/10.1007/BF02468653

17. Cho H, Nagata N, Shimbo T, Sakurai T, Sekine K, Okubo H, Imbe K, Watanabe K, Mikami S, Yokoi C, Kobayakawa M, Mizokami M, Yanase M, Akiyama J, Uemura N (2016) Recurrence and prognosis of patients emergently hospitalized for acute esophageal variceal bleeding: a long-term cohort study. Hepatol Res 46(13):1338-1346. https://doi.org/10.1111/hepr.12692

18. Zang L, Sun Z, Li W, Liu X (2015) Meta-analysis of risk factors of gastroesophageal varices rebleeding after therapeutic endoscopy. Zhonghua Gan Zang Bing Za Zhi 23(4):275-280. https://doi.org/10.3760/ cma.j.issn.1007-3418.2015.04.009

19. Abbasi A, Butt N, Bhutto AR, Munir SM (2010). Correlation of thrombocytopenia with grading of esophageal varices in chronic liver disease patients. J Coll Physicians Surg Pak; 20(6):369-372, DOl: 06.2010/ JCPSP.369372.

20. Sigal SH, Sherman Z, Jesudian A (2020) Clinical implications of thrombocytopenia for the cirrhotic patient. Hepat Med 12:49-60. https://doi. org/10.2147/HMER.S244596

21. Elkenawy YN, Elarabawy RA, Ahmed LM, Elsawy AA (2020) Portal vein flow velocity as a possible fast noninvasive screening tool for esophageal varices in cirrhotic patients. JGH Open 4(4):589-594. https://doi.org/10.1002/ jgh3.12301

22. ShaStri M, KulKarni S, Patell R, Jasdanwala S (2014) Portal Vein Doppler: a tool for non-invasive prediction of esophageal varices in cirrhosis. J Clin Diagn Res 8(7):MC12-MC15. https://doi.org/10.7860/JCDR/2014/8571.4589

23. Xu L, Ji F, Xu QW, Zhang MQ (2011) Risk factors for predicting early variceal rebleeding after endoscopic variceal ligation. World J Gastroenterol 17(28): 3347-3352. https://doi.org/10.3748/wjg.v17.i28.3347

24. Mostafa EF, Mohammad AN (2014) Incidence and predictors of rebleeding after band ligation of oesophageal varices. Arab J Gastroenterol 15(3-4):135141. https://doi.org/10.1016/j.ajg.2014.10.002

25. Sameea EA, Omar N, Gabal AA, Waked I, Saleh SM (2005) Predictors of early rebleeding following endoscopic control of variceal bleeding. Am J Gastroenterol 100:S118. https://doi.org/10.14309/00000434-200509001-00289

26. Nasrullah A, Kashif M, Nasrullah H, Siddiqu AM (2020) Risk factors associated with early variceal rebleed after endoscopic variceal band ligation in cirrhotic Hepatitis B and C patients with variceal bleed. J Fatima Jinnah Med Univ 14(1):30-33. https://doi.org/10.37018/PENC3048

27. Simonetto DA, Liu M, Kamath PS (2019) Portal hypertension and related complications: diagnosis and management. Mayo Clin Proc 94(4):714-726. https://doi.org/10.1016/j.mayocp.2018.12.020

\section{Publisher's Note}

Springer Nature remains neutral with regard to jurisdictional claims in published maps and institutional affiliations. 